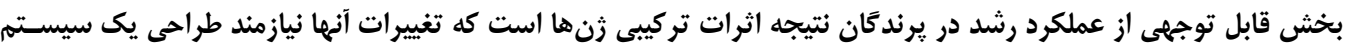

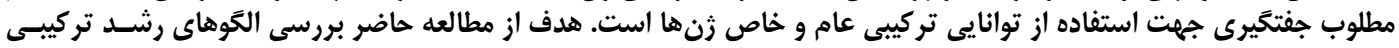

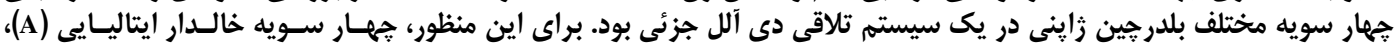

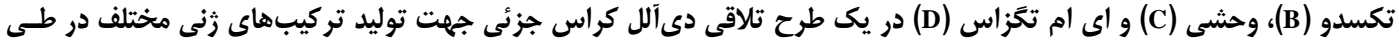

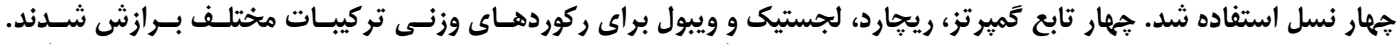

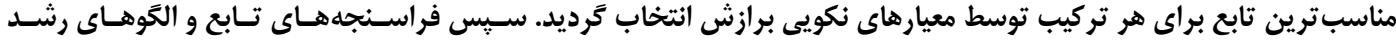

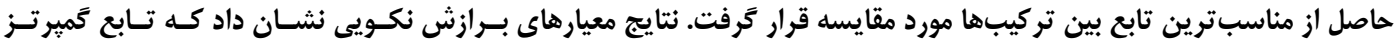

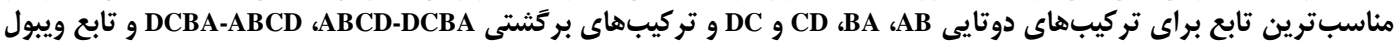

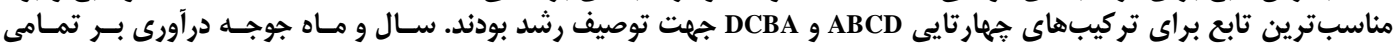

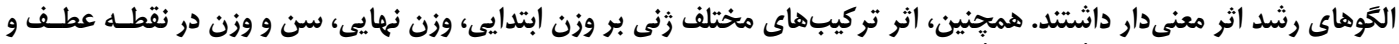

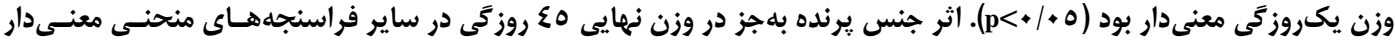

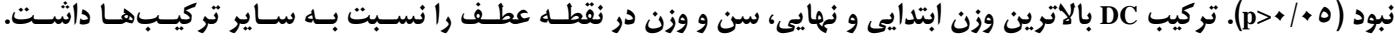

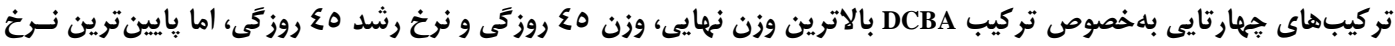

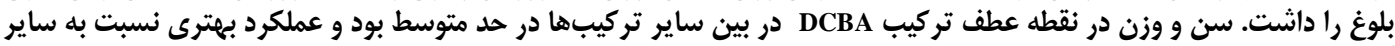
آميختهها داشت كه مى تواند بهدليل بيشترين مقدار هتروزيس در اين اين تركيب باشد.

وازههاى كليدى: آميخته تَرى، بلدرجين زاينى، توابع رشد، كميرتز

يكى ديكر از مزاياى توصيف منحنى رشد محسوب مى شود و

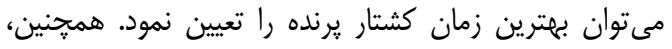

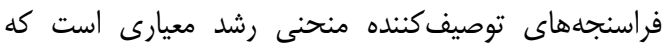

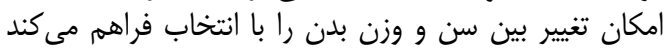

(1).19)

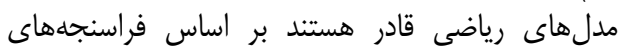

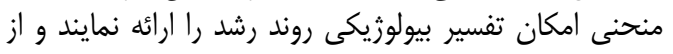

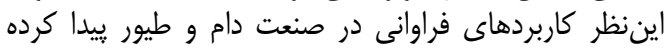

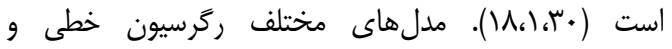

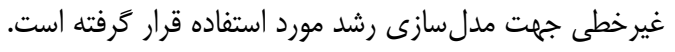

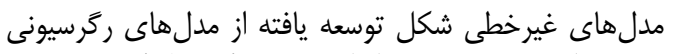

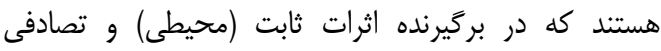

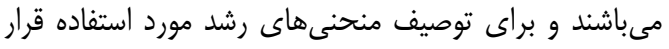

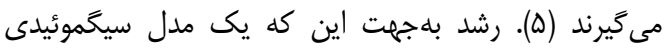

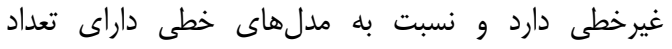

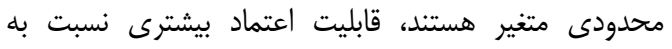

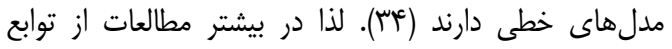

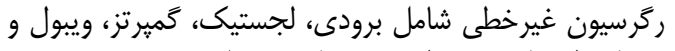

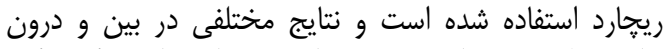

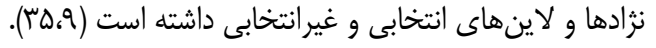

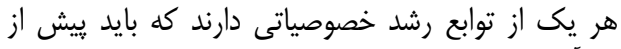

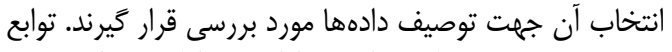
رشد كميرتز و لجستيك داراى شكل سيخموئيدى ترايع
مقدمه

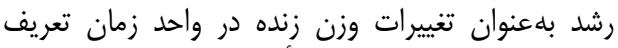

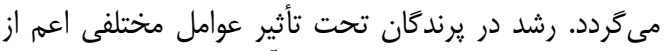

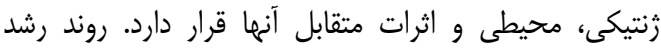

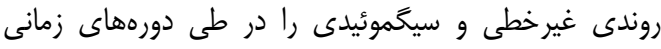

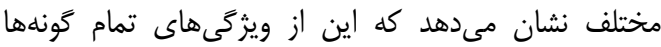

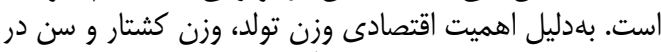

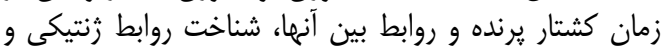

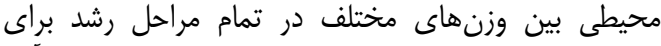

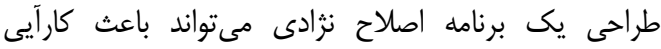

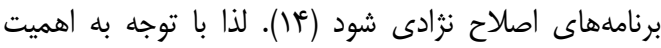

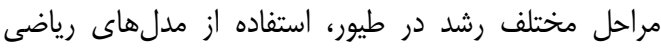

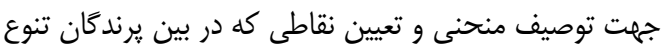

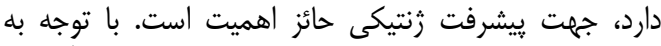

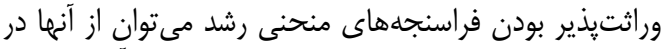

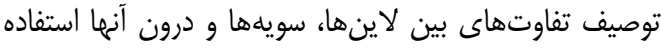

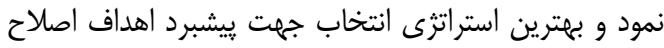

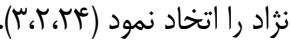

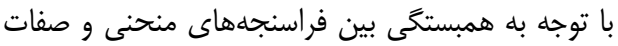

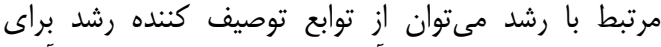

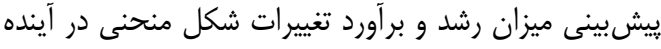

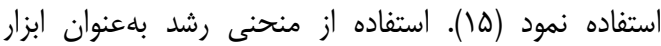

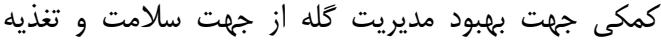


كه صرفاً با اتخاد سيستمهاى جفتخيرى مناسب در بين

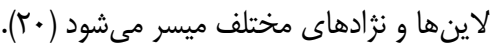

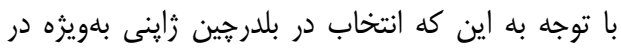

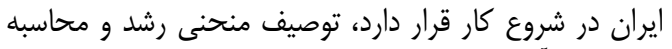

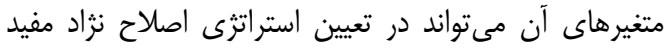

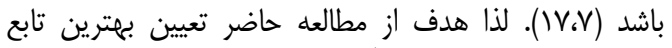

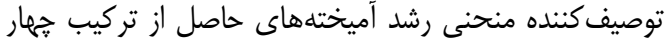

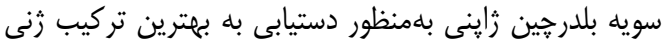

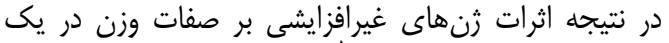

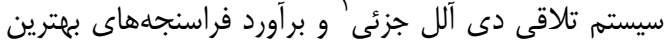

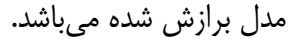

\section{مواد و روشها موردا جمعيت مورد مطالعه}

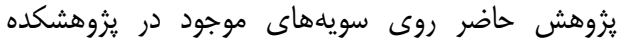

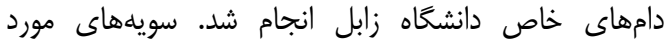

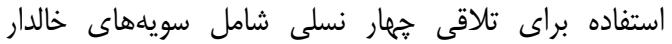

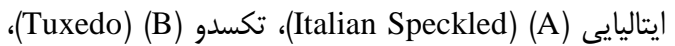

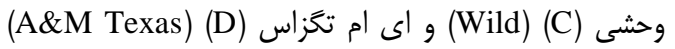

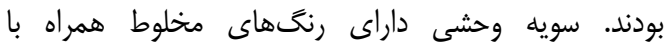

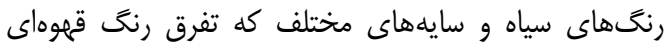

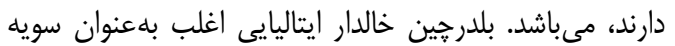

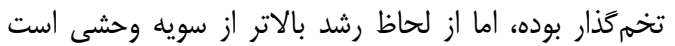

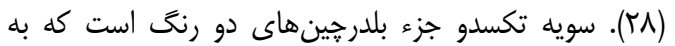

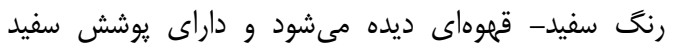

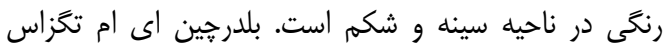

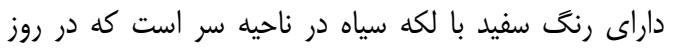

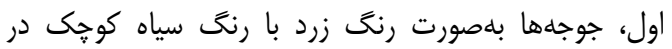

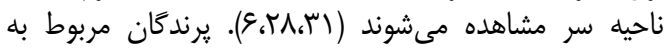

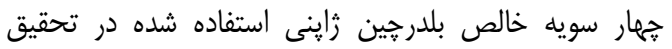

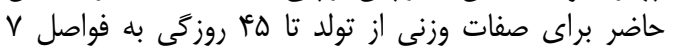

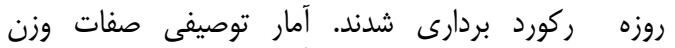

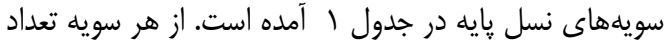

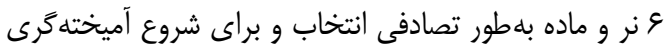

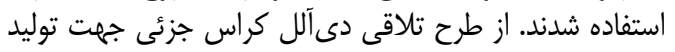

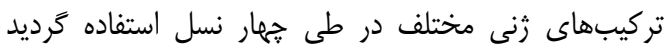

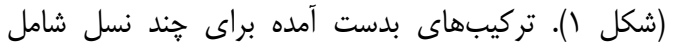
ABCD-DCBA ،DCBA ،ABCD ، DC ، CD ،BA ، AB

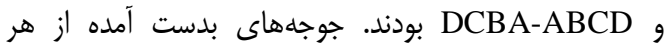

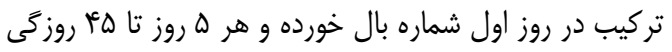

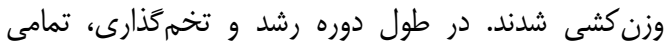

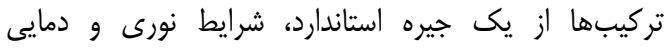
يكسانى برخوردار بودند.

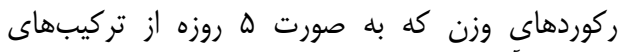

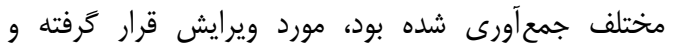

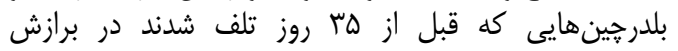

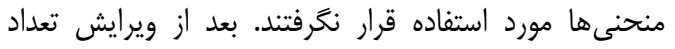

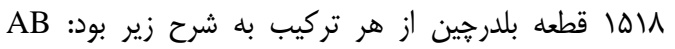

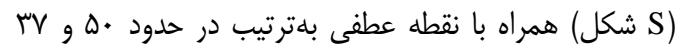

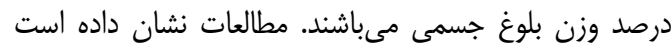

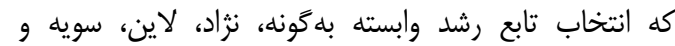

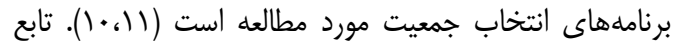

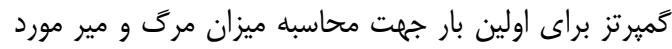

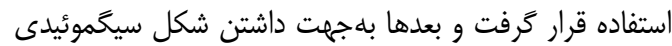

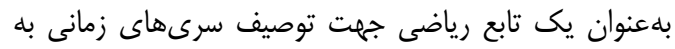

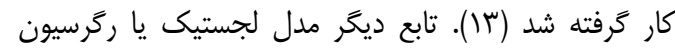

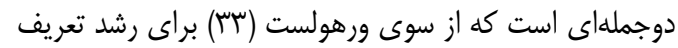

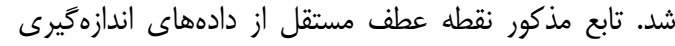

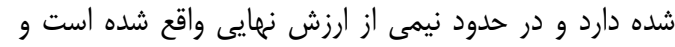

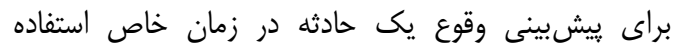

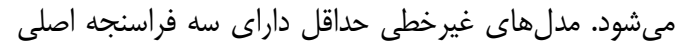

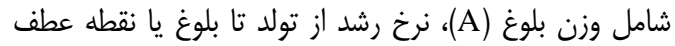

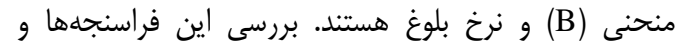

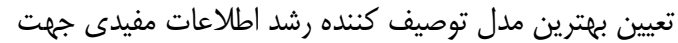

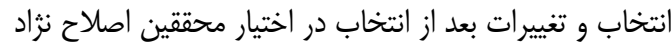

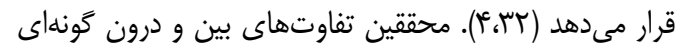

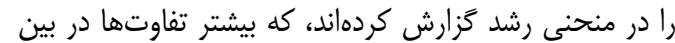

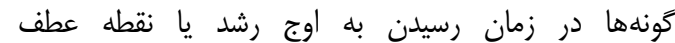
منحنىهاى رشد است. ريكلف (צاء

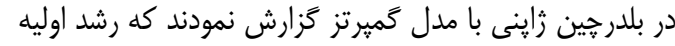

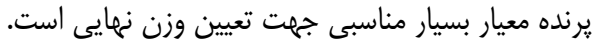

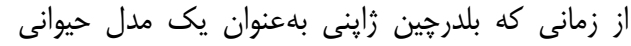

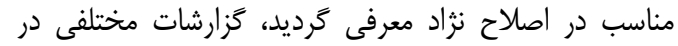

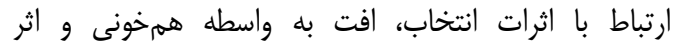

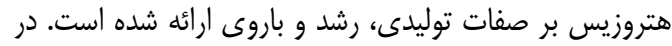

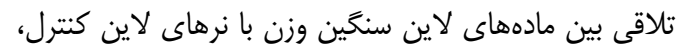

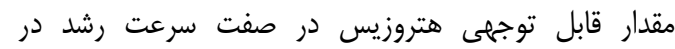

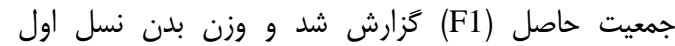

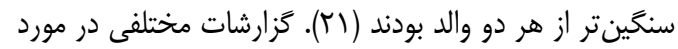

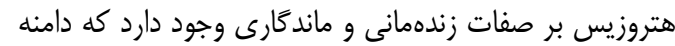

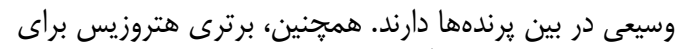

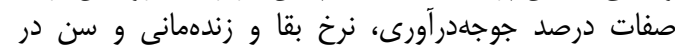

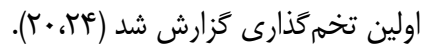

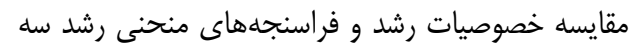

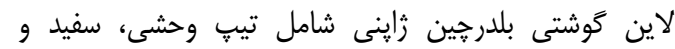

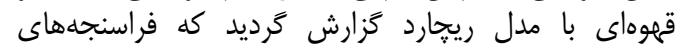

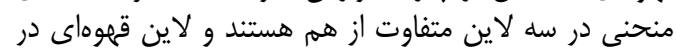

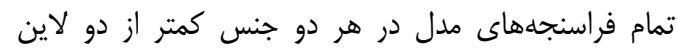

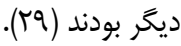
حداكثر راندمان در توليد طيور در نتيجه اثرات تركيبى دريى

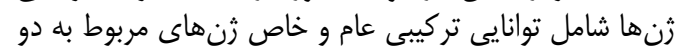

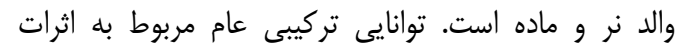

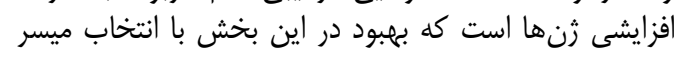

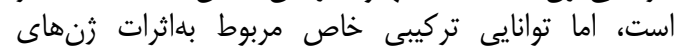

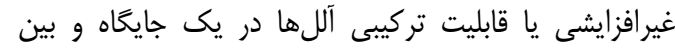

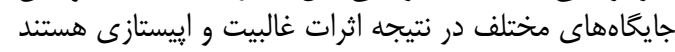

1- Partial di-allele cross 
از معيارهاى برازش نكويى ذيل براى تركيبهاى مختلف

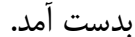
ضريب تبيين تصحيح شده $R_{\text {Adj }}^{2}=1-\left[\left(\frac{n-1}{n-p}\right) \times\left(1-R_{\text {model }}^{2}\right)\right]$ ميانخين مربعات خطا $M S E=\frac{S S E}{n-p}$

$$
\text { معيار اطلاعات آكائيك }
$$

$B I C=n \cdot \ln \left(\frac{S S E}{n}\right)+p \cdot \ln (n)$ معيار اطلاعات بيزى معيأ

در معيارها، $R_{\text {model }}^{2}$

1-(SSE/SST)

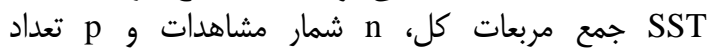

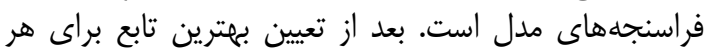

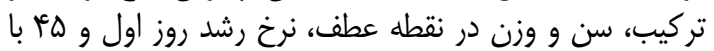

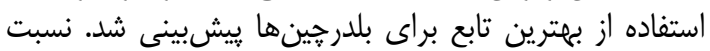

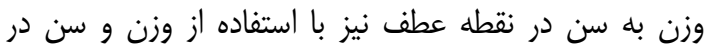

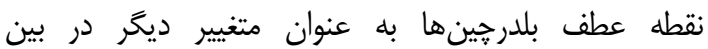

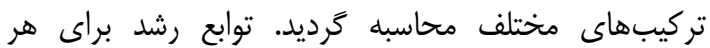

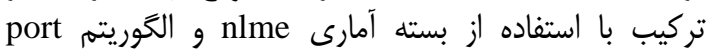

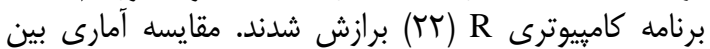

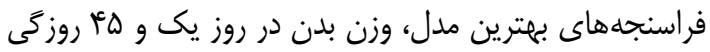

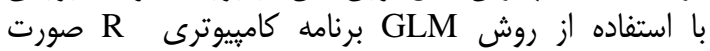
كرفت.
$\mathrm{ABCD}$ (Ira) DC (IFT) CD (ITY) BA (ITV) و (rDF) ABCD-DCBA (IVA) DCBA (I $(Y \mathrm{~V})$ DCBA-ABCD

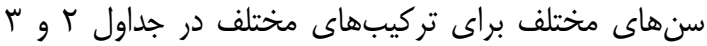
آورده شده است.

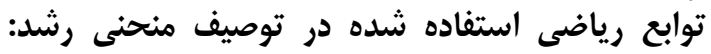

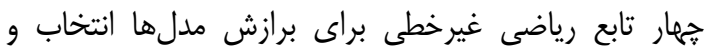

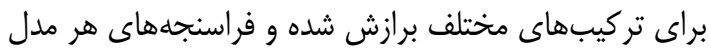

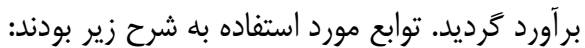

تابع كميرتز (T)

$W=W_{0} \exp \left\{[1-\exp (-k \times A g e)] \ln \left(\frac{W_{f}}{W_{0}}\right)\right\}$

تابع ريجارد (Tه)

$$
W=\frac{W_{0} W_{f}}{\left[W_{0}^{m}+\left(W_{f}^{m}-W_{0}^{m}\right) e^{-k t}\right]^{1 / m}}
$$

$$
W=\frac{W_{0} W_{f}}{\left[W_{0}+\left(W_{f}-W_{0}\right) \exp (-k \times A g e)\right]}
$$

تابع ويبول (عَ)

$W=W_{f}-\left(W_{f}-W_{0}\right) \exp \left[-(k \times A g e)^{m}\right]$

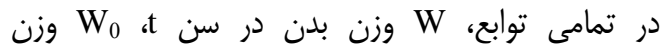

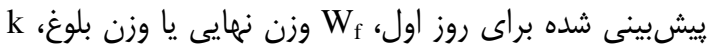
شاخص بلوغ و m فراسنجه مدل مى باشيد.

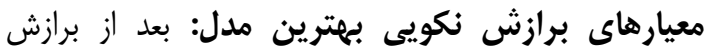
تمامى توابع براى تركيبهاى مختلفي بهرئ بهترين تابع با استفاده 
جدول ا- آمار توصيفى وزن بلن در هفتههاى مختلف براى جهار سويه اصلى Table 1. Descriptive statistics of body weight in different weeks for four major strains

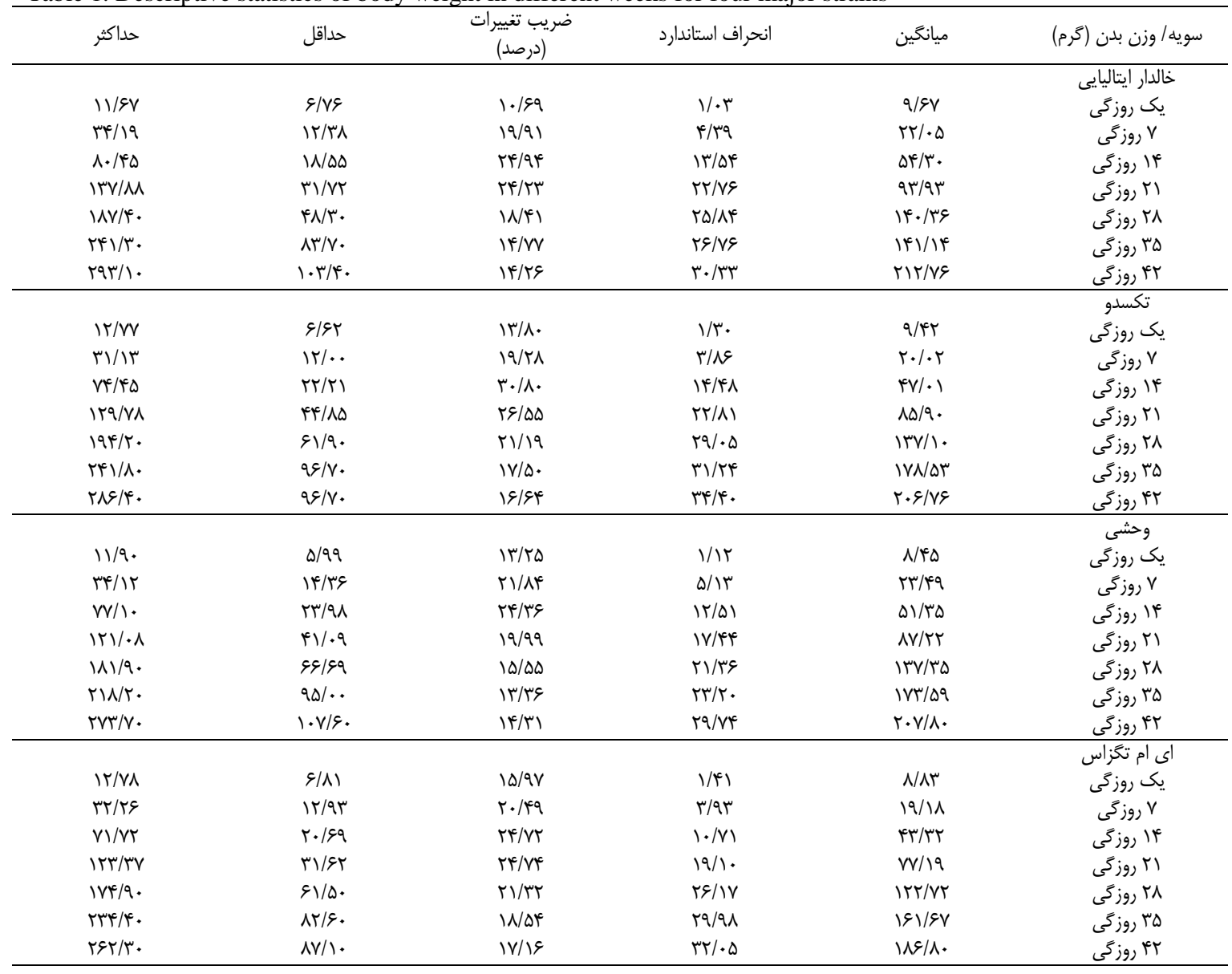

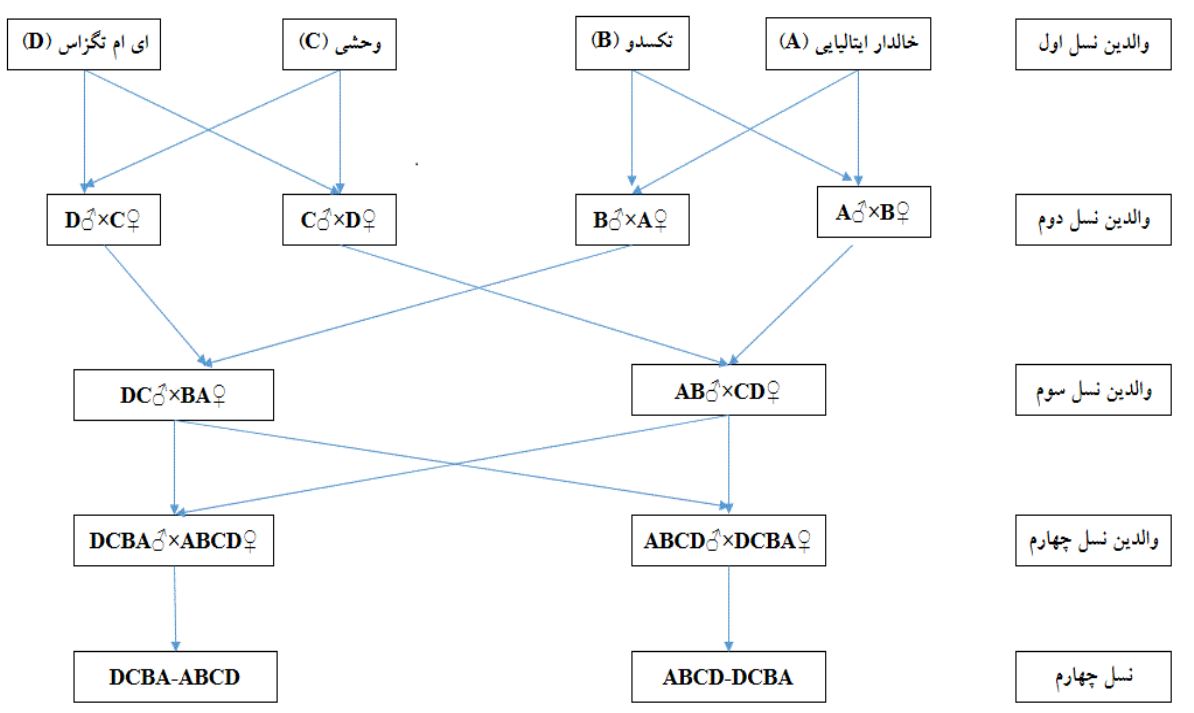

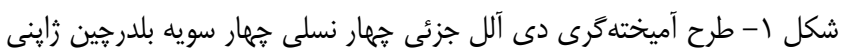

Figure 1. Partial di-allele cross breeding design of four Japanese quail strains in four generations. 
جدول r- آمار توصيفى وزن بلن در روزهاى مختلف (كرم) آميختهاى دوتايى سويههاى مختلف بلدرجين زإِنى

Table 2. Descriptive statistics of body weight in different days (in gram) for dual-crossbred of

\begin{tabular}{|c|c|c|c|c|c|c|}
\hline حداكثر & حداقل & ضريب تغييرات & انحراف استاندارد & ميانگين & وزن بدن (كرم) & تركيب \\
\hline $1 . / \%$ & $9 / 91$ & $11 / \wedge 9$ & $1 / \cdot 4$ & $\Lambda / V \Delta$ & يك روزىى & \multirow{10}{*}{$\mathrm{AB}$} \\
\hline$T V / q)$ & $11 / T \Delta$ & $\mid \mathrm{N} / \cdot \mathrm{r}$ & $r / 91$ & $r . / . r$ & ه روزگى & \\
\hline$\Delta T / \digamma A$ & $r r / r F$ & $r r / v$. & N/א & $r v / I r$ & •ا روزىى & \\
\hline $1 / 98$ & rা/rq & re/VG & $19 / 49$ & $81 / 81$ & ها روزگى & \\
\hline $11 \cdot / T V$ & $|c| s$. & $M I / V F$ & $1 N / 1 Q$ & $\Lambda \uparrow / \uparrow \wedge$ & •r روزگى & \\
\hline $\mid G T / \cdot r$ & $\Delta V / \Delta 1$ & $r q / 1 \Lambda$ & $r r / \cdot 1$ & $1.9 / 99$ & هז روزى & \\
\hline$r \cdot r / Q Q$ & $q \cdot / r 1$ & $\mathrm{rI} / 9 \mathrm{~V}$ & זו/אז & IFE/TK & • بر روزى & \\
\hline$T E \cdot / Q T$ & $\| V / \wedge \Delta$ & IN/r. & Tr/Tळ & IVE/Vq & هז روز & \\
\hline TET/KT & $\mid V E / V \Lambda$ & ع & $T V / \Delta \Lambda$ & $r \cdot g(\Delta)$ & 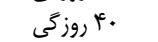 & \\
\hline$T V F / \Delta \Lambda$ & IVq/To & $11 / 98$ & rE/IV & $T M E / T r$ & ه广 روز & \\
\hline $11 / \Upsilon A$ & S/DS & $I D / I V$ & $1 / \Gamma$ & $\mathrm{N} / \mathrm{VV}$ & يك روزىى & \multirow{10}{*}{ BA } \\
\hline re/r. & $\mid r / G \Delta$ & $19 / 4 r$ & $r / v 1$ & $19 / 1$. & ه روزى & \\
\hline$\Delta F / A F$ & 19/9D & $r \in / \notin q$ & $q / \cdot r$ & & •ا روزگى & \\
\hline$q . / v T$ & $r F / \Lambda V$ & $r \Delta / r q$ & $19 / \pi T$ & $94 / 1 \pi$ & ها روزگى & \\
\hline $1.8 / 91$ & $9 \cdot / k^{c}$ & $\mid V / \cdot F$ & $1 \% / 91$ & $\Lambda T / \cdot r$ & •r روزى & \\
\hline IDT/FT & $V r / V{ }^{c}$ & $r r / 4 q$ & $r \Delta / T \Lambda$ & $1 \cdot V / 81$ & هז روز & \\
\hline $\mid \Lambda \Delta / \wedge V$ & $1 . r / / F^{f}$ & $\mid V / 19$ & $T Y / Q V$ & $\mid f r / v q$ & •r روزگى & \\
\hline TMF/T. & $\mid r \cdot / 80$ & $\left.|Q| \cdot\right|^{c}$ & $r \varepsilon / F A$ & $|V \varepsilon| \cdot r$ & هז روزگى & \\
\hline & $\mid 9 \cdot / V 1$ & $1 . / T r$ & $r \cdot / \Delta r$ & $r \cdot \cdot|\Lambda|$ & 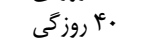 & \\
\hline TGY/AV & $|\varepsilon| / R \varepsilon$ & $11 / \wedge \Delta$ & rq/.. & $r / 9 / \mu^{\prime}$ & ه广 روز & \\
\hline $1 . / V 1$ & 9/99 & $q / \uparrow \wedge$ & $\cdot / 1 / 9$ & $q / \cdot V$ & يك روزىى & \multirow{10}{*}{ CD } \\
\hline$r q / 19$ & $\mid r / F$. & IV/TA & $r / 98$ & TI/Tr & ه روزگى & \\
\hline$\Delta V / A$. & $\Gamma \Psi / \Lambda I$ & & $9 / \mu$. & $r / \Delta \mu$ & •ا روزگى & \\
\hline $9 \% / F$ & $r r / \cdot r$ & q & $1 \Delta / V \Delta$ & $q V / q T$ & ها روزگى & \\
\hline $11 \omega / \Gamma$. & FI/א & $19 / 4 V$ & IV/GT & $q \cdot / \Delta \omega$ & •r روزگى & \\
\hline$|\varepsilon \mu / \cdot|$ & $V T / V$. & TE/Tr & $\mathrm{rV} / \mathrm{VV}$ & $\| f / M$ & هז روزگى & \\
\hline$r \cdot \Delta / \Delta \Delta$ & ११/VV & זT/אז & & $|0 \cdot / \pi|$ & •r روزى & \\
\hline TME/T. & $11 \cdot / \Delta \Delta$ & ع & $r \cdot / \pi r$ & $|x+/ n|$ & هז روزگى & \\
\hline rQN/צG & $\mid r \Delta / v \Delta$ & $\mid r / \cdot r$ & $r \Delta / \Delta$. & $r \mid r / \cdot V$ & 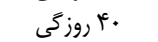 & \\
\hline$r . g / g r$ & $189 / \lambda f$ & $I T / r T$ & $T N / 1 D$ & ו וא/חז & ه广 روز & \\
\hline $11 / 91$ & G/QT & $\mid \omega / \cdot 1$ & $1 / \Gamma$ & $\Lambda / \Lambda \mathcal{A}$ & يك روزىى & \multirow{10}{*}{ DC } \\
\hline$r Q / \Delta)$ & $11 / \cdot 1$ & $r \Delta / \kappa$. & سו/D & $r \cdot / r$. & ه روزىى & \\
\hline$\Delta \Delta / 11$ & $\mid N / T V$ & $\mathrm{TV} / \cdot \mathrm{V}$ & 1.1 .4 & $r V / \cdot r$ & •ا روزگى & \\
\hline 9.189 & $r r / r$. & $r r / l q$ & $19 / 9$. & $9 \cdot 1 M$ & ها روزگى & \\
\hline $\mid K F / 1$. & ع & $r q / a t$ & Tr/N & $\Lambda \cdot 1 \cdot \Delta$ & • r روزى & \\
\hline$|Q V / D|$ & $\Gamma \varphi / Q \Delta$ & $r q / \varphi_{0}$ & TN/FD & 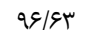 & هז روزى & \\
\hline $19 \% / \wedge \&$ & $V I / r$. & TV/G. & $r V / T V$ & $\mid r \Delta / \cdot \varphi^{f}$ & •r روزىى & \\
\hline$r r q / r$. & १৭/^৯ & & كז/ו & $199 / 44$ & هז روزگى & \\
\hline TET/*1 & $\mid T \Delta / r \Delta$ & $|N / \mu|$ & rs/9q & $r \cdot . / r \Lambda$ & 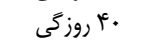 & \\
\hline$r \Lambda 1 / 9 \Delta$ & $|f| / r$. & IS/AF & $r v / 11$ & $r r \cdot / r$. & ه广 روزى & \\
\hline
\end{tabular}


جدول ؟ـ- آمار توصيفى وزن بدن در روزهاى مختلف (بر حسب گرم) آميختهاى جهارتايى و تركيبهاى برگشتى سويههاى مختلف

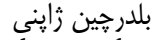

Table 3. Descriptive statistics of body weight in different days (in gram) for fourth crossbred and its reciprocal combination of different strains of Japanese quail

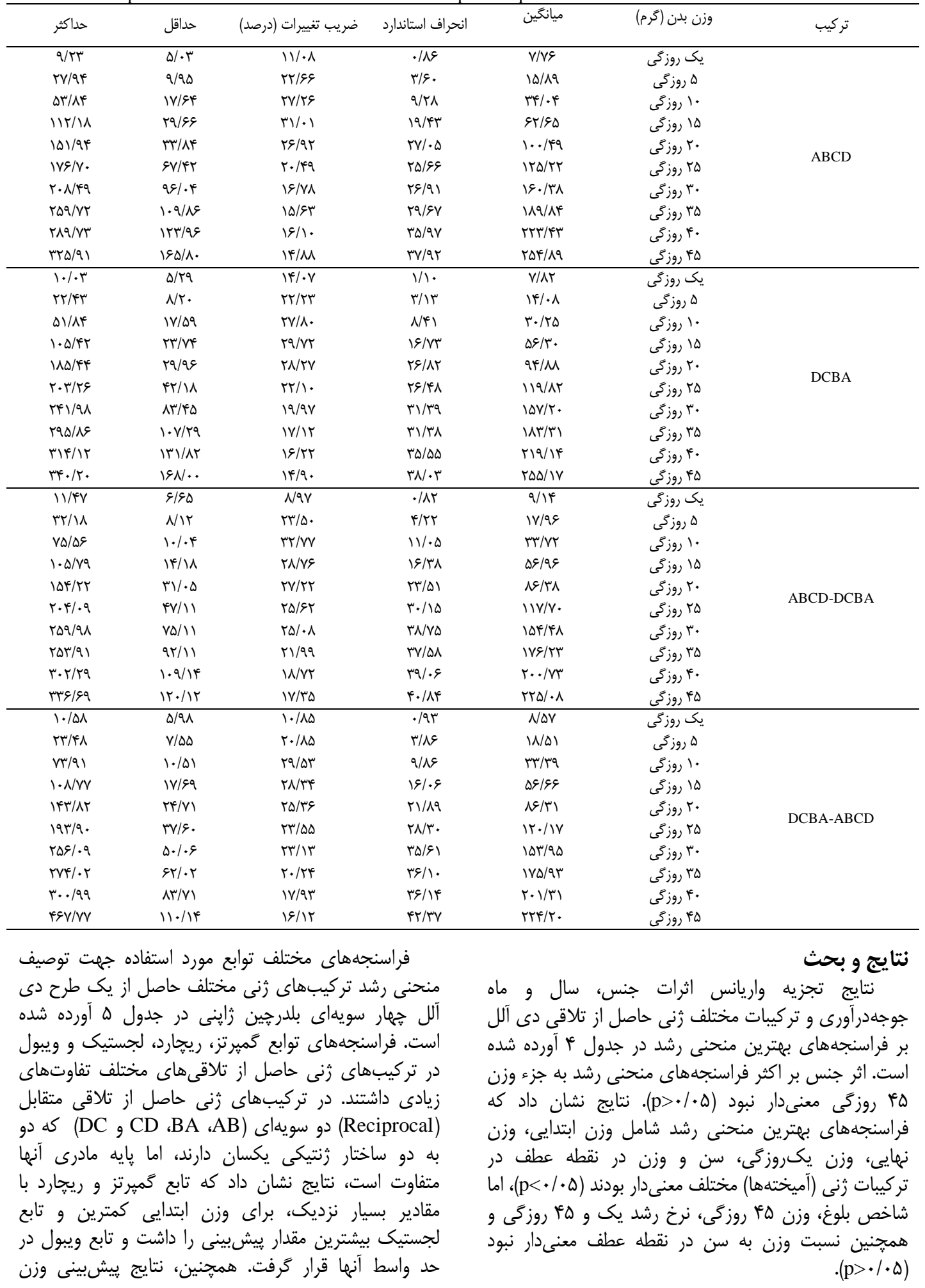



برازش منحنىهاى رشد در آميختههاى دى آلل بلدرجين زاينى و مقايسه الكوهاى رشد آميختهها ...

تركيبهاى دو سويالى شامل نتايج متفاوتى بود، به نحوى كه به

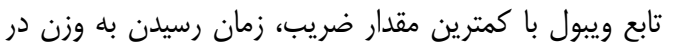

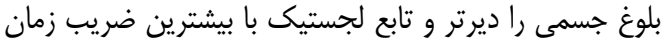

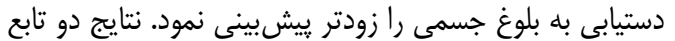

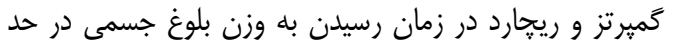
واسط قرار داشت.
نهايى با توابع مختلف در تركيبهاى دو سويهاى تابع ويبول

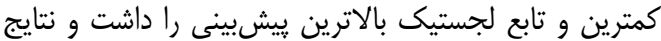

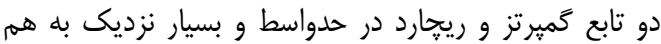

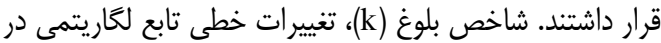

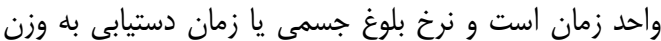

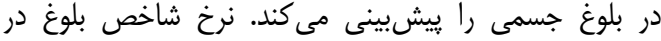

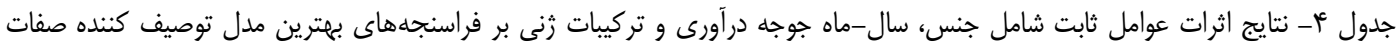

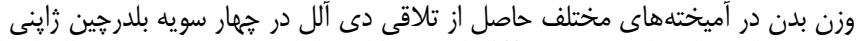

Table 4. The results of fixed effects including sex, year-month hatching and different crossbred on the parameters of best model describing body weight traits in different crossbred from diallele cross design of four Japanese quail strains

\begin{tabular}{|c|c|c|c|}
\hline تركيب ثنى & سال - ماه جوجهدرأورى & جنس ج جن & فراسنجه* \\
\hline.$/$ TM & $\cdot / \cdots 1$ & . /ITVG & وزن ابتدايى \\
\hline 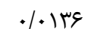 &. $\mid \ldots 1$ & rוt. & ل وزن نهايى \\
\hline 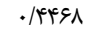 &.$\ldots r$ & $\cdot /$ N N & شاخص بلوغ \\
\hline.$/ \ldots 1$ &.$|\ldots|$ & - MEFA & وزن يك روزگى \\
\hline 1999. &.$/ \ldots 1$ &.$/ \cdots 1$ & وزن هأ روزىى \\
\hline 的. &.$/ \ldots 1$ & . /areq & سن در نقطه عطف \\
\hline $.1 \cdot 1 \cdot 9$ &.$/ \ldots 1$ & 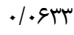 & وزن در نقطه عطف \\
\hline . &.$/ \ldots 1$ & $\cdot / T \Delta \Lambda \mid$ & نسبت وزن به سن در نقطه عطف \\
\hline - & $\% \circ \mathrm{\Delta V}$ & $\cdot / \Lambda \cdot \Delta F$ & نرخ رشد ا روزگى \\
\hline .10919 &.$\ldots r$ & $\cdot / 19 \cdot v$ & نرخ رشد ها روزگ \\
\hline
\end{tabular}

جدول ه- فراسنجههاى توابع مختلف در آميختههاى حاصل از طرح دى آلل در جهار سويهاى بلدر خين زاينى Table 5. Parameters of different growth cures in crossbred populations from di-allele cross design in

\begin{tabular}{|c|c|c|c|c|c|}
\hline $\mathrm{m}(\mathrm{SE})$ & $\mathrm{W}_{\mathrm{f}}(\mathrm{SE})$ & k (SE) & $* \mathrm{~W}_{0}(\mathrm{SE})$ & منحنى & تركيب \\
\hline- & $\Gamma \Delta \cdot / \backslash \varphi(\Gamma \backslash / \Gamma \Delta \cdot)$ & $\cdot / \cdot \Delta(\cdot / \cdot \cdot \Delta)$ & $9 / \cdot(I / A r I)$ & كَمِيرتز & \multirow{4}{*}{$\mathrm{AB}$} \\
\hline$\cdot / 10(\cdot / 4 \backslash 9)$ & TrY/IT (qr/qYI) & $.1 .9(. / . Y F)$ & $9 / 91(5 / 1+1)$ & 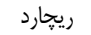 & \\
\hline- & rq $/ 9 \cdot(11 / \cdot \Lambda \cdot)$ & $.11 \cdot(\cdot 1 \cdot .9)$ & $\mid F / .9(1 / q \pi T)$ & لجستيك & \\
\hline $1 / V^{c}(\cdot / r \cdot 1)$ & $\operatorname{MYN} / \operatorname{ME}^{\prime}(\Lambda \cdot / \Delta \cdots)$ &.$/ . r(\cdot / \cdot . \Delta)$ & $1 . / 90\left(\Gamma / \Lambda F F^{4}\right)$ & وييول & \\
\hline- & 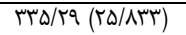 & $\cdot / \cdot \Delta(\cdot / \cdot \cdot \varphi)$ & $q / \cdot r(1 / q \times 1)$ & كميرتز & \multirow{4}{*}{ BA } \\
\hline$\cdot / \cdot \Lambda(\cdot / \mu \& Y)$ & TrI/F. $(9.1 .81)$ & $.1 . \Delta(\cdot / . r I)$ & $Q / \Delta \Lambda(T / A T F)$ & ريجارد & \\
\hline- & $r \Delta F / \cdot \wedge(q / F \Delta F)$ & $\cdot 11 \cdot(\cdot 1 \cdot .9)$ & $14 / 1 \cdot(1 / 491)$ & لجستيك & \\
\hline $\mathrm{I} / \mathrm{v} \cdot(\cdot / \mathrm{\Lambda} \cdot)$ & TFT/TF (VT/M•) &.$/ . r(\cdot / \cdot . \Delta)$ & $1 . / \Gamma q(\Gamma / F V \varepsilon)$ & ويبول & \\
\hline- & ror/rq (rr/q..) & $\cdot / \cdot \Delta(\cdot / \cdot \bullet r)$ & $1 . / \Delta S(I / F \wedge \Delta)$ & كميرتز & \multirow{4}{*}{$\mathrm{CD}$} \\
\hline$-\cdot / 1 \cdot(\cdot / r \Lambda F)$ & $r V V / r g(V N / T \cdot V)$ & $.1 .4(.1 .19)$ & $9 / V q(Y / q T I)$ & ريجارد & \\
\hline - & TEN/G9 (N/FAF) & $\cdot / 1 \cdot\left(\cdot / \cdot r^{\prime}\right)$ & $10 / Q Y(1 / T r \cdot)$ & لجستيك & \\
\hline $1 / 09(\cdot / 1<q)$ & $\varphi \cdot \Delta / V \Lambda(q \vee / \& \Delta \cdot)$ &.$/ . r(. / .0 \Delta)$ & $1 . / \Delta F(r / 1 \Delta Q)$ & ويبول & \\
\hline- & $F I V / \Delta D(\varepsilon N / I r I)$ & $.1 .4(.1 . .9)$ & $1 . / V^{e}(T / F T \Lambda)$ & كميرتز & \multirow{4}{*}{$\mathrm{DC}$} \\
\hline$-* / * r(\cdot / r q \Delta)$ & FIN/DF (KTN. . I) &.$/ . F(\cdot / \cdot r F)$ & $1 . / V T(F / / T \Delta)$ & ريجارد & \\
\hline - & $r V V / V^{e}(19 / 4 \Delta \cdot)$ & $.1 .9(\cdot 1 \cdot . v)$ & $10 / .1(T / .99)$ & لجستيك & \\
\hline $1 / \Delta F(\cdot / T q 1)$ & GNN/FG (AF/AT.) &.$/ .1(\cdot / \cdot 14)$ & $1 . / \Delta T(\Delta / / F \&)$ & ويبول & \\
\hline- & 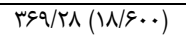 & $\cdot / \cdot \Delta(\cdot / \cdot \cdot r)$ & $\mathrm{V} / \Lambda \mathrm{(I//QT)}$ & كميرتز & \multirow{4}{*}{$\mathrm{ABCD}$} \\
\hline.$- / 4 \Gamma(\cdot / / V q)$ & $\Delta r F / \Delta F(I F V / q T \cdot)$ &.$/ . r(. / .11)$ & $r / \mathcal{T V}(r / \mu / F)$ & ريجارد & \\
\hline- & TME/VD (V/\&SV) &.$/ 11(\cdot / \cdot .4)$ & $I F / F \cdot(I / I I V)$ & لجستيك & \\
\hline 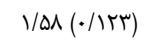 & EAT/T) (1../q..) &.$/ . r(. / .+r)$ & $\Delta / \kappa^{*} \cdot(\Gamma / \Lambda \Delta \cdot)$ & ويبول & \\
\hline- & $r M N / I \Delta(r \backslash / \& \Delta \cdot)$ & $\cdot / \cdot \Delta(\cdot / \cdot \cdot r)$ & $\mathrm{V} / \cdot\left(\mathrm{I} / \mathrm{V} / \varphi^{\mathrm{T}}\right)$ & كَمِيرتز & \multirow{4}{*}{ DCBA } \\
\hline.$- / F r(\cdot / I V Q)$ & DQT/AT (IQF/rMA) &.$/ . r(. / .11)$ & $r / . \cdot(r / I f V)$ & ريجارد & \\
\hline- & $r q 1 / 9 V(\Lambda / \cdot r r)$ &.$/ 11(. / . * 4)$ & $\mid r / r \cdot(1 / \cdot+\cdot)$ & لجستيك & \\
\hline $1 / 94(\cdot / 1 r q)$ & rqs/vq (Irr/A..) &.$/ .4(. / . \cdot c)$ & $\Delta / I T(K / \& \wedge F)$ & ويبول & \\
\hline- & $\Gamma \cdot \Gamma / q^{\mu}(\Lambda / \cdot \cdot \cdot)$ & $.1 .9(. / . .4)$ & $8 / 4 \wedge(\cdot / 9+9)$ & كميرتز & \multirow{4}{*}{ ABCD-DCBA } \\
\hline$\cdot / 1 \Delta(\cdot / 1 F \Delta)$ & $r \Lambda V / T H(I Q / I F \cdot)$ &.$/ \cdot v(\cdot / . \cdot 9)$ & $V / \Delta T(I / \backslash A V)$ & ريجارد & \\
\hline- & $r \in \Psi / A T(\Gamma / \Gamma \cdot \Delta)$ &.$/ 1(\cdot / . r)$ & $1 T / 10(\cdot / 819)$ & لجستيك & \\
\hline $1 / 9 \cdot(\cdot / \cdot \vee q)$ & TYN/D. $(I T / \Delta) \cdot)$ &.$/ . r(. / . .1)$ & $q / q \pi(1 / F V V)$ & وييول & \\
\hline- & TQN/FV $(N / T \cdot 1)$ & $.1 .9(\cdot / . . r)$ & $g / K F(\cdot / g \vee \Lambda)$ & كميرتز & \multirow{4}{*}{ DCBA-ABCD } \\
\hline ./ $Q(\cdot / \Lambda \Delta \Psi)$ & rNT/ז\& (ID/.1.) &.$/ \cdot v(\cdot 1 \cdot .9)$ & $V / T r(1 / T \Delta F)$ & ريجارد & \\
\hline- & TFI/DS (T/KT.) & ./Ir $(\cdot / \cdot r)$ & $11 / 98(\cdot / 9 \Delta 8)$ & لجستيك & \\
\hline $1 / 91(\cdot / \cdot \Lambda \cdot)$ & $r V r / r F(11 / r q \cdot)$ &.$/ . r(. / . .1)$ & $9 / V^{N}(1 / \Delta V Y)$ & وييول & \\
\hline
\end{tabular}




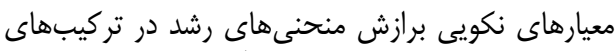

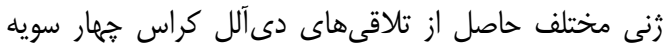

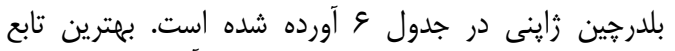

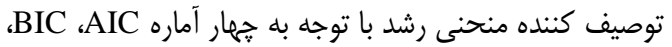
تعيين كرديد. تابعى كه بلهوريه

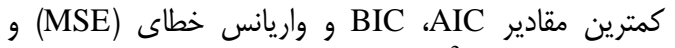

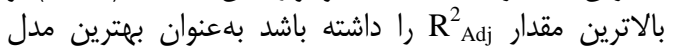

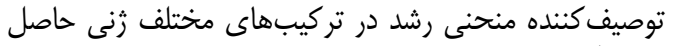

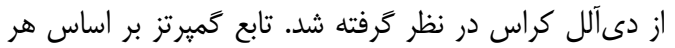

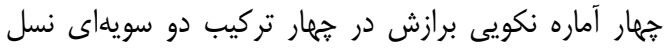

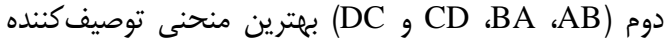
رشد بود، اما در تركيبهاى جهار نزادى نسل سوم (DBCD) (DCBA

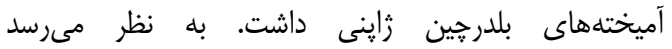

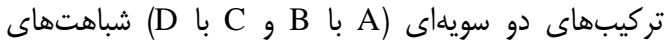

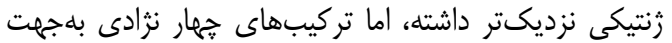

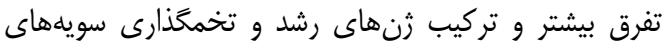

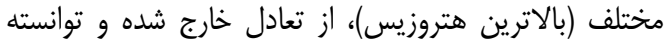
توصيف متفاوتى از منحنى رشد ارائه نمايند. در تلاقيى دى دي آلل

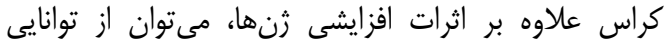

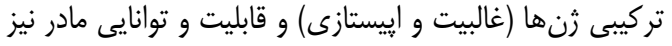

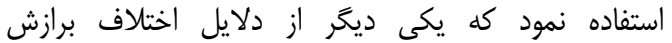

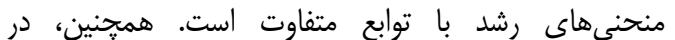

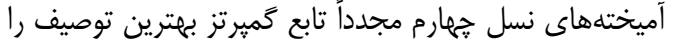

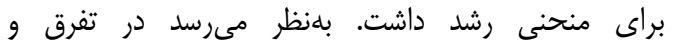

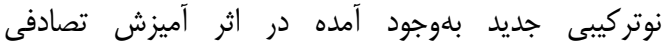

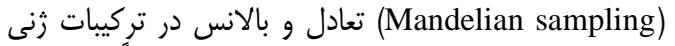

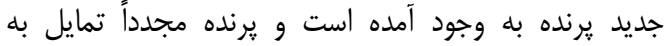

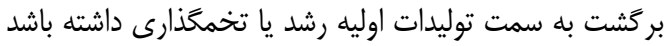
(كاهش هتروزيس).
نتايج فراسنجههاى توابع مختلف در آميختههاى تهار سويهاى نسل سوم شامل ABCD (نتاج حاصل تاقلاقى نرهاى

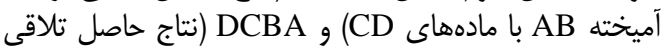

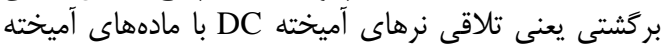

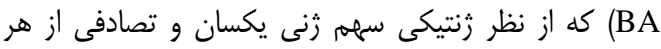
سويه را دريافت نمودهاند ( وزن إئد

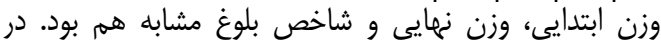

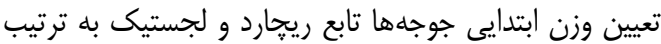

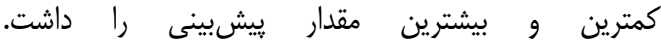

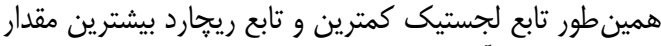

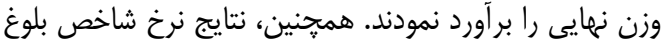

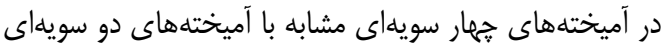

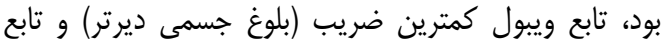

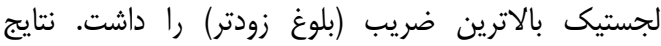

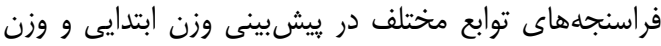

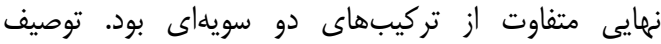

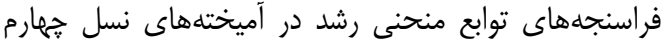

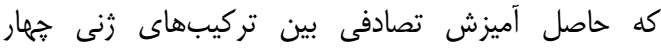

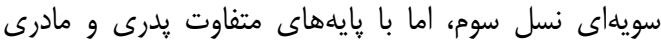

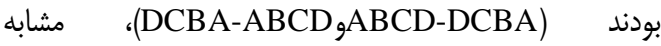
آميختههاى دو نزادى نسل دوم بود. توابع گميرتز و لجستيك

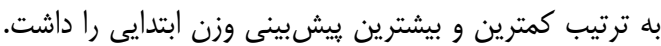

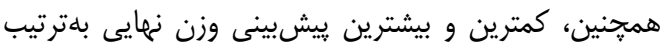

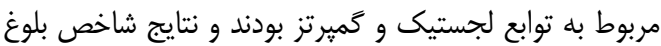

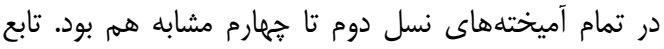

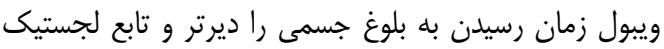

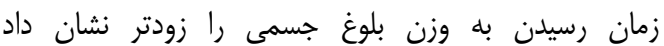

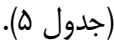



برازش منحنىهاى رشد در آميختهاى دى آلل بلدرجين زاينى و مقايسه الكوهاى رشد آميختهها ...

جدول צ- معيارهاى نكويى برازش منحنىهاى رشد براى آميختهاى مختلف دى آلل كراس در جهار سويه بلدرجين زاينى Table 6. Goodness of fit criterions of growth cures for different crossbred from di-allele cross design in four

\begin{tabular}{|c|c|c|c|c|c|}
\hline وييول & لجستيك & ريجارد & كميرتز & معيار" & تركيب \\
\hline TEFT/TE & THET/KG & TFFI/Iq & rerq/r. & AIC & \multirow{4}{*}{$\mathrm{AB}$} \\
\hline TFq./T. & $T F \Delta \Delta / V T$ & rEQQ/IF & TFAT/GG & BIC & \\
\hline $91 / \% \wedge$ & $91 / \% \wedge$ & 91/ & $91 / \% 4$ & $\mathrm{R}_{\mathrm{Adj}}^{2}$ & \\
\hline $0|q / F|$ & $019 / 81$ & $\Delta I V / T V$ & DID/GT & MSE & \\
\hline$\Gamma+\cdot / \Delta$. & $T / . T / F T$ & 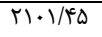 & $r .99 / 49$ & AIC & \multirow{4}{*}{ BA } \\
\hline rाlq/q. & $r \mid l g / r \Delta$ & TIN/AD & $r|| r / F i$ & BIC & \\
\hline$q \pi /<q$ & $q \Psi / F V$ & $q T / \Delta T$ & $q \Psi / \Delta \Delta$ & $\mathrm{R}_{\mathrm{Adj}}^{2}$ & \\
\hline$r G 4 / 19$ & 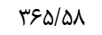 & rEY/G. & זו/וצr & MSE & \\
\hline TVMN/IS & TVQ)/QA & rVAS/QS & $r \gamma \wedge \Delta / \cdot \Delta$ & AIC & \multirow{4}{*}{ CD } \\
\hline ץ.N/rq & $r \Lambda \cdot v / v^{c}$ & $r \Lambda \cdot v / \downarrow q$ & $(\mu \cdot 1 / r)$ & BIC & \\
\hline $9 T / T V$ & $9 T / 14$ & $q T / r q$ & $9 \% / \pi)$ & $\mathrm{R}_{\text {Adj }}^{2}$ & \\
\hline FVy/q1 & |N|l/qu & $F V D / \Delta F$ & $F V \in / \Delta$. & MSE & \\
\hline$T \mu G \Delta / \Delta 1$ & rrgr/vq & rMgr/A. & THGT/A. & AIC & \multirow{4}{*}{$\mathrm{DC}$} \\
\hline & $r \mathrm{rW} / \mathrm{Ar}$ & 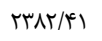 & rrVg/Aq & BIC & \\
\hline$\Delta V / F$. & $N V / F F$ & $N V / F F$ & $\Delta V / F q$ & $\mathrm{R}_{\mathrm{Adj}}^{2}$ & \\
\hline$V r^{\prime} \omega / \mu$ & V & r/אח & $V \pi \cdot / r q$ & MSE & \\
\hline qr.D/A. & $9 \times 4 / / 9$ & $9 \pi .9 / \pi 1$ & $9 \pi \cdot 9 / \wedge \Delta$ & AIC & \multirow{4}{*}{$\mathrm{ABCD}$} \\
\hline GTKN/rK & GTDQ/TI & GTKN/VD & GKTV/AV & BIC & \\
\hline 91/^r & १1/\% & $91 / \wedge \mu$ & १)/WV & $\mathrm{R}_{\mathrm{Adj}}^{2}$ & \\
\hline $9 \mid 9 / 11$ & $9 \Delta F / F \Delta$ & 95.119 & GKF/Fq & MSE & \\
\hline $99 \mathrm{Vr} / 19$ & $V \cdot 1.1 / \alpha^{4}$ & $99 V / \Delta r$ & $99 \mathrm{~W} / \mathrm{TI}$ & AIC & \multirow{4}{*}{ DCBA } \\
\hline $9990 / 91$ & $V \cdot Y N / q$. & $9998 / 91$ & $9990 / 8 \wedge$ & BIC & \\
\hline $91 / 44$ & $91 / \cdot 1$ & $91 / 45$ & 91/ץ & $\mathrm{R}_{\mathrm{Adj}}^{2}$ & \\
\hline$q \times q / F V$ & 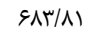 & $90.1 . r$ & $9 \Delta F / 1$. & MSE & \\
\hline$r f \mid r q / \Lambda 1$ & $T F|Q| / \& q$ & $T F I T N / F 4$ & $T F I T V / \Delta V$ & AIC & \multirow{4}{*}{ ABCD-DCBA } \\
\hline$r \in I \Delta q / .$. & TFIVE/AF & TFIOV/GT & $T F 10 . / 9 T$ & BIC & \\
\hline NV/FT & NV/RT & $N V / F F$ & $\Lambda V / F T$ & $\mathrm{R}_{\text {Adj }}^{2}$ & \\
\hline VaN $/ \cdot \Delta$ & N.D/R & VQV/gr & VqV/दq & MSE & \\
\hline $191 \cdot N / T F$ & $19 / T V / T$. & $\mid 91.8 / Q T$ & $191 \cdot 0 / 99$ & AIC & \multirow{4}{*}{ DCBA-ABCD } \\
\hline |س/r/ & $|9|<q / 90$ & $191 \pi f / 8$. & $191 T N / 11$ & BIC & \\
\hline$M / \Gamma \Delta$ & $M / T F$ & $M / r q$ & $M / \mu q$ & $\mathrm{R}_{\mathrm{Adj}}^{2}$ & \\
\hline VTN/TV & $V \Gamma \Delta / \uparrow \Lambda$ & $V T V / 99$ & $V T V / V$. & MSE & \\
\hline
\end{tabular}

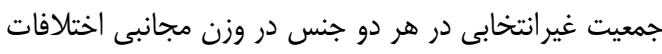

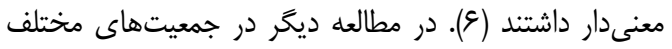

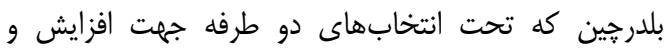

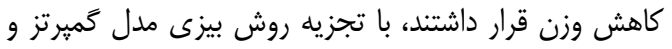

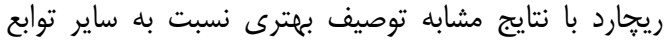

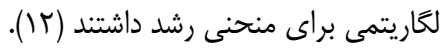

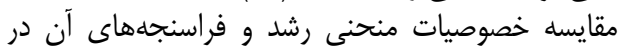

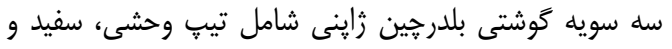

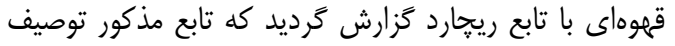

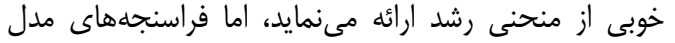

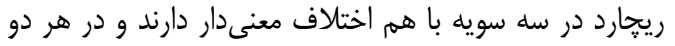

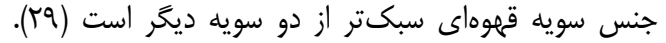

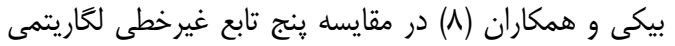

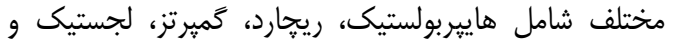

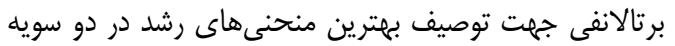

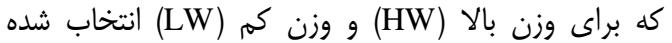

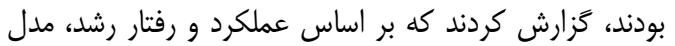

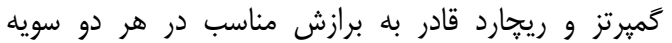

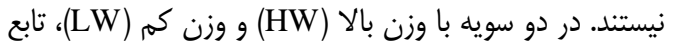

مدلهاى رشد اطلاعات مورد نياز جهت فهم زيديدههاى

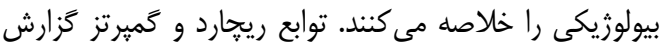

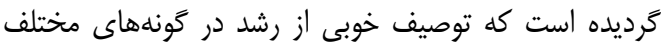

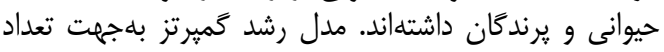

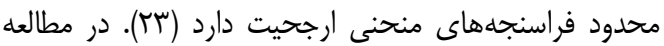

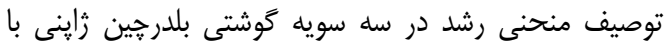

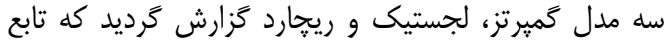

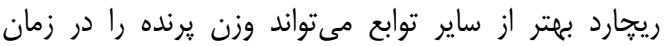

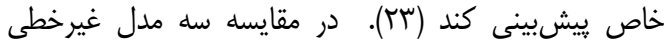

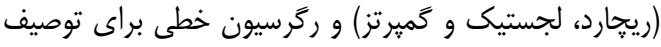
دادههاى رشد، مدل خطى ضعيفترين توصيف رادئ را در مقايسه

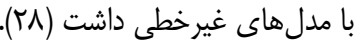

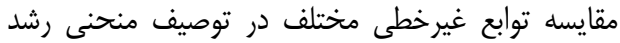

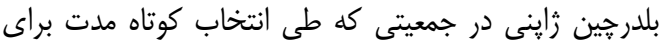

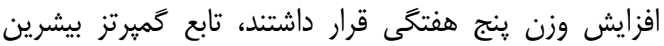

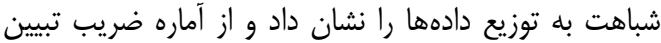

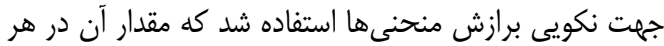

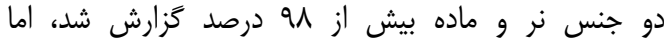
فراسنجههاى منحنى در بهترين تابع در بين لاين انتخابى با لان 


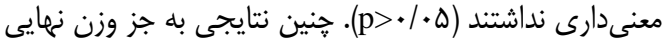

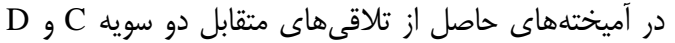

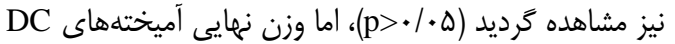

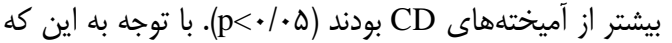

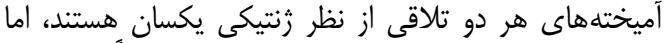

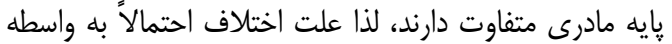

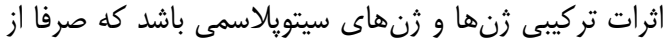
والد ماده به نتاج منتقل مى شعوند.
ريجارد مناسبترين برازش را داشت. لذا نتايج تحقيقات مشابه

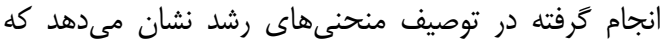

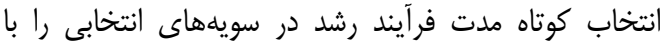

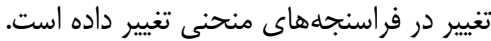

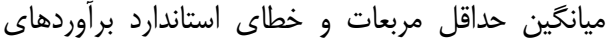

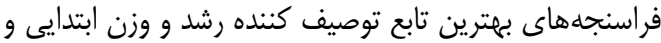

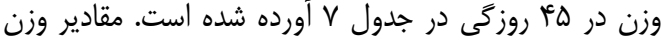

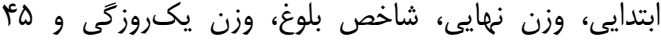

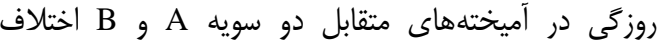

جدول V- ميانگين حداقل مربعات و خطاى استاندارد فراسنجه هاى تابع منحنى رشد، وزن يك و هأ روزگى براى تركيب هاى مختلف Table 7. Least square means and standard error of growth curve function parameters, weight at hatching and 45 days

\begin{tabular}{|c|c|c|c|c|c|}
\hline وزن هt روزگى & وزن يك روزگى & شاخص بلوغ & وزن نهايي & وزن ابتدايى & تركيب \\
\hline$T Y Y / T V Y(V / G V \cdot)^{0}$ & N/VQT $(\cdot / / \Lambda))^{\mathrm{aDCC}}$ & $\cdot / \cdot \psi \wedge(\cdot / \cdot \cdot r)^{0}$ & 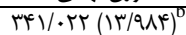 & $\Lambda / \Lambda \cdot Y\left(\cdot / V^{r} \Lambda\right)^{a}$ & $\mathrm{AB}$ \\
\hline$r / q / r V \Lambda ~(V / Q \Lambda \mu)^{D}$ & N/VVG $(\cdot / 19 Y)^{\mathrm{DC}}$ &.$/ .+q(\cdot / \cdot . r)^{\mathrm{D}}$ & $r \Delta \cdot / \Lambda \Delta T(I F / \Lambda \mu r)^{D}$ & 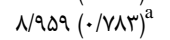 & $\mathrm{BA}$ \\
\hline & $q / \cdot V \Delta(\cdot / / F \Delta)^{\mathrm{ab}}$ &.$/ * \psi \wedge(\cdot / \cdot r)^{\mathrm{D}}$ & $r \Delta q / q 19(11 / r / r)^{D}$ & $1 . / 090(\cdot / 094)^{a}$ & CD \\
\hline Tr./TQS (V/ATr $)^{\mathrm{D}}$ & $\Lambda / \Lambda \& \Delta(\cdot / / M \Lambda)^{\text {abc }}$ & $\cdot / \cdot \psi \cdot\left(\cdot / \cdot r^{\prime}\right)^{\mathrm{D}}$ & $F \cdot \Delta / F T T(I F / \Delta T Y)^{a}$ & $1 . /$ \& & DC \\
\hline$r \Delta F / \wedge 9 \Delta(F / \Lambda \mid r)^{\mathrm{a}}$ & $V / V G \Delta(\cdot / / 1 \Delta)^{a}$ &.$/ \cdot r r(\cdot / \cdot r)^{c}$ & $r / q q / F \cdot r(\Lambda / \Lambda V V)^{b}$ & $8 / 4 \cdot q(\cdot / 48 q)^{b}$ & $\mathrm{ABCD}$ \\
\hline$r \Delta \Delta / I V \varepsilon(\kappa / \Delta \uparrow \varepsilon)^{\mathrm{a}}$ & $V / N) \&(\cdot / 1.9)^{a}$ &.$/ \cdot r r(\cdot / \cdot r)^{c}$ & $r q r / \cdot V)(\Lambda / r q \cdot)^{D}$ & $\Delta / V \Lambda \mathcal{}(\cdot / F+r)^{\mathrm{D}}$ & DCBA \\
\hline$t r A / \cdot N t(t / 4 \& Q)^{D}$ & $q / \operatorname{lec}(\cdot / \cdot \Delta q)^{a}$ &.$/ \cdot \Delta \wedge(\cdot / \cdot .1)^{\mathrm{a}}$ & $r \cdot \Delta / \Delta \Delta F(\mathcal{F} / \Delta \Delta q) C$ & $\operatorname{s/gVV}(\cdot / T+1)^{\mathrm{D}}$ & ABCD-DCBA \\
\hline$T / Y / T+1(T / Y M q)^{D}$ & $N / D F V(.1 .99)^{c}$ & $.1 .0 \mathrm{Q}(\cdot / \cdot .1)^{\mathrm{a}}$ & 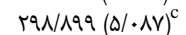 & G/rqA $(. / / q q)^{\mathrm{D}}$ & DCBA-ABCD \\
\hline
\end{tabular}

ABCD

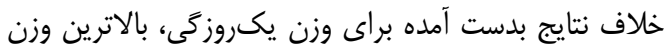

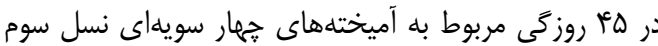
بCBA و ABCD

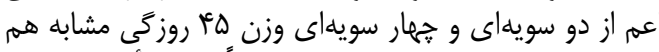

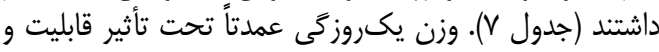

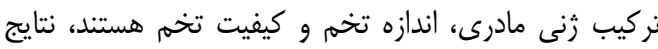

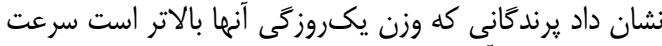

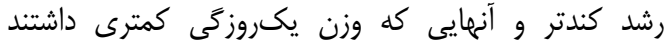

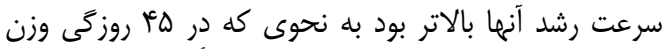

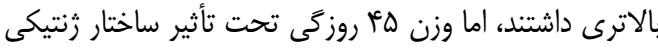

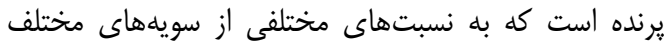

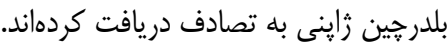

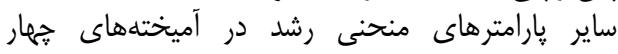

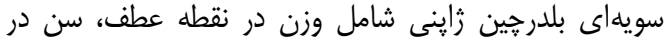

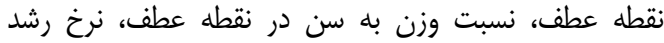

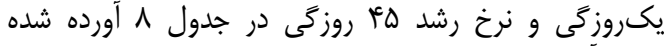

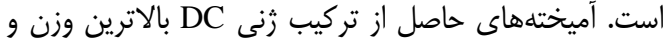

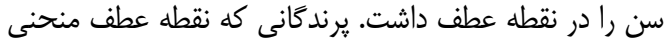

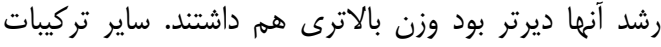

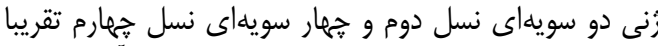

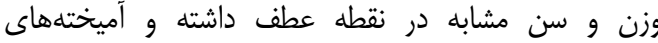

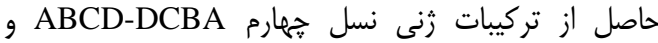

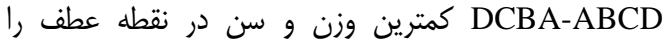

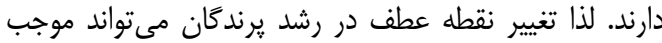

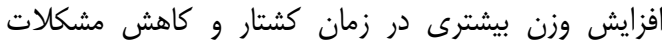
فيزيولوزيكى و متابوليكى در يرندكان گَردد (اسّ).
فراسنجههاى منحنى رشد شامل وزن ابتدايى، وزن نهايی،

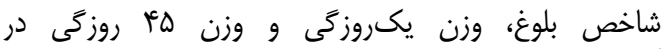

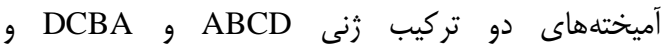

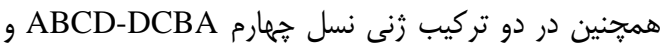
DCBA-ABCD

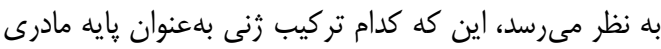

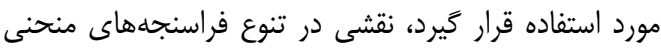

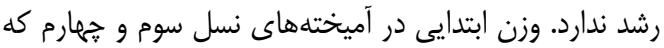

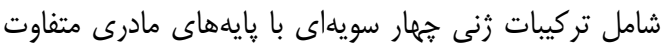

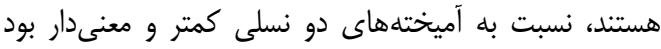

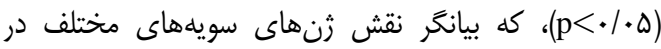

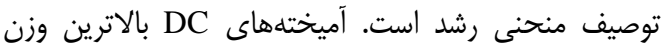

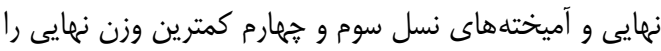

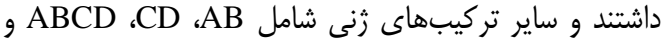

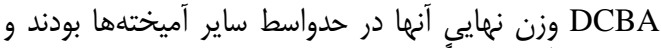

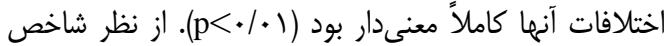

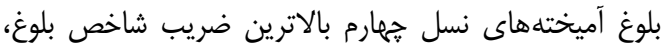

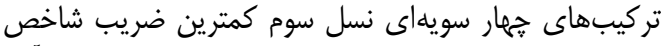

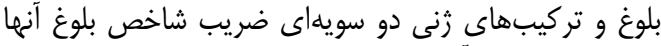

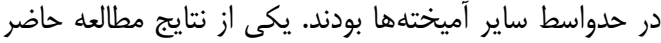

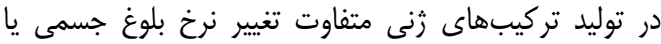

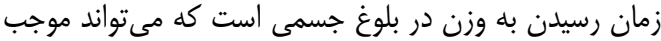

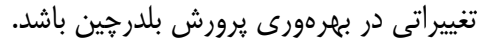

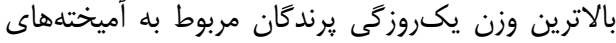

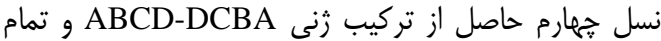

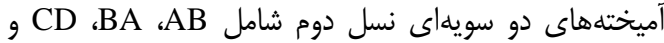

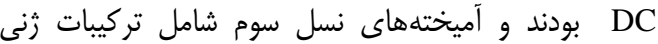


$1 T \cdot$ برازش منحنىهاى رشد در آميختهاى دى آلل بلدرجين زاينى و مقايسه الكوهاى رشد آميختها ...

جدول ^- ميانگَين حداقل مربعات و خطاى استاندارد فراسنجههاى منحنى رشد شامل وزن و سن در نقطه عطف و نرخ رشد يك و هأ

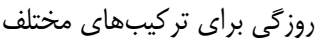

Table 8. Least square means and standard error of curve function parameters including weight and age at inflection point and growth rate of one and 45 days for different crossbred

\begin{tabular}{|c|c|c|c|c|c|}
\hline نرخ رشد ه广 روزگى & نرخ رشد يك روزگى & نسبت وزن به سن نقطه & سن در نقطه عطف & وزن در نقطه عطف & تركيب \\
\hline$r / g r \Lambda(\cdot / \Gamma) \cdot)^{\mathrm{DC}}$ & I/DT. $(\cdot / I I I)^{c}$ & 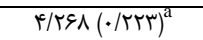 & $r F / \backslash \Delta \Lambda(T / V \cdot F)^{D}$ & $\mid r \Delta / \kappa \Delta \omega(\Delta / / \Delta \Delta)^{D}$ & $\mathrm{AB}$ \\
\hline F/Trt $(\cdot / \text { MTr })^{\text {ad }}$ & $1 / \Delta \Delta F(\cdot / I 1 \Lambda)^{D C}$ & $\kappa / \Delta \Lambda \cdot(\cdot / r r q)^{a}$ & $r q / \Delta 11(r / N \& \Lambda)^{\text {Dca }}$ & $|T N / \Lambda| F(\Delta / \& \& V)^{D}$ & BA \\
\hline$F / \Lambda Q F(\cdot / / F T)^{\text {ad }}$ & $1 / 089(\cdot / .19)^{c}$ & $\operatorname{F/VG\Lambda }(\cdot / / \vee \Lambda)^{\mathrm{a}}$ & $r q / \& \& q(r / \Lambda \& \Lambda)^{\text {Dca }}$ & D & $\mathrm{CD}$ \\
\hline 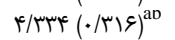 & I & F/Rr $(\cdot / R T I)^{a}$ & $\kappa e / r+.(r / \Lambda) \cdot)^{a}$ & $\mid \mp q / 1 \neq \varepsilon(\Delta / r \Delta V)^{a}$ & DC \\
\hline$f / 19 \cdot(\cdot / 194)^{\mathrm{ad}}$ & $\cdot / M r(\cdot / \cdot v \cdot)^{a}$ & $f / 4 \varphi g(\cdot / / 41)^{a}$ & $r \cdot / \varepsilon \Lambda \cdot(I / V) \varepsilon)^{\mathrm{DC}}$ & ITT/TFA $(T / T V T)^{D}$ & $\mathrm{ABCD}$ \\
\hline$r / \Delta r \varepsilon(\cdot / M \Lambda)^{a}$ &.$/ W 1(.1 .99)^{\mathrm{a}}$ & $r / \kappa q \varepsilon(\cdot / / r r)^{a}$ & $r \cdot / r r \cdot(1 / 9 T r)^{D C}$ & $\mid r N / \cdot V \in(T / \cdot q T)^{D}$ & DCBA \\
\hline$r / T E T(\cdot / 1 . . \cdot)^{c}$ & $1 / A) V(\cdot / . r \varepsilon)^{a}$ & $\kappa / \Delta \cdot V(\cdot / \cdot V r)^{a}$ & $r V / \Delta D Q(\cdot / M \Lambda T)^{\mathrm{Ca}}$ & $\| r / F \cdot V(Y / q \Lambda))^{c}$ & ABCD-DCBA \\
\hline$r / r) \cdot(\cdot / M r)^{c}$ & $1 / V \& V(\cdot / \cdot F \cdot)^{\text {ad }}$ & $F / \Delta \Delta F(\cdot / \cdot 1))^{\mathrm{a}}$ & $r \& / / 81(. / 914)^{a}$ & $1.9 / 9 \Delta 9(1 / A \vee \Delta)^{c}$ & DCBA-ABCD \\
\hline
\end{tabular}

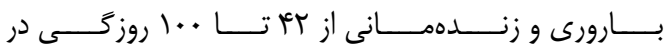

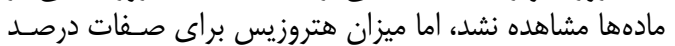

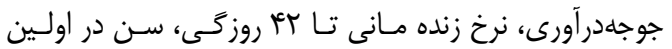

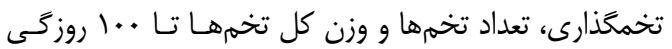

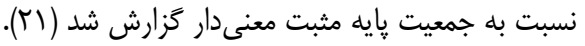

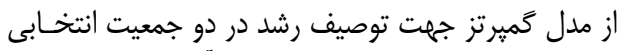

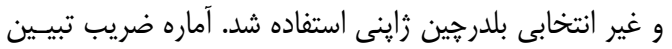

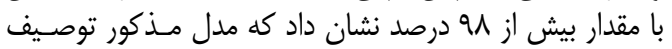

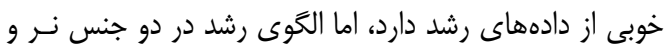

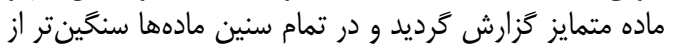

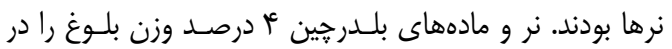

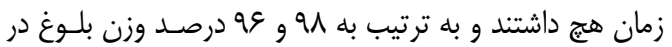

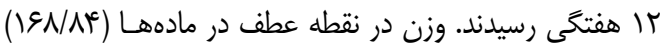

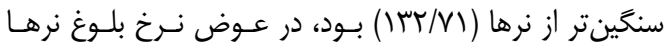

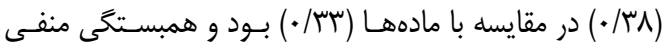

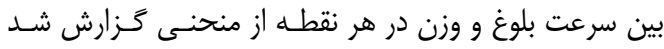

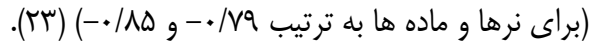

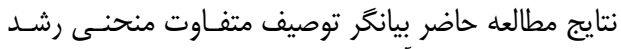

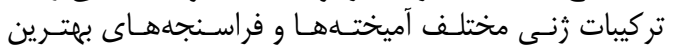

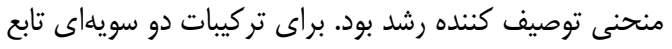

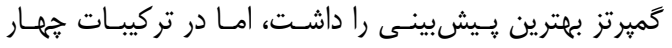

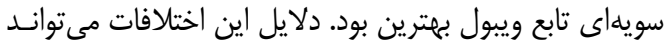

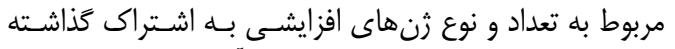

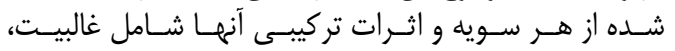

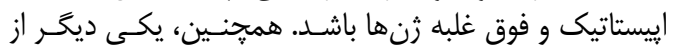

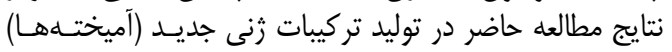

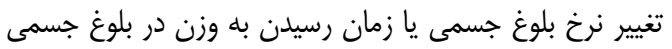

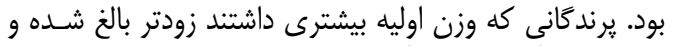

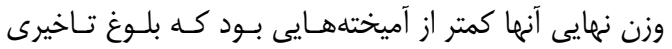

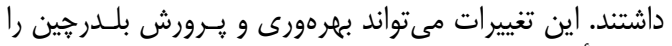
تحت تأثير قرار دهد.

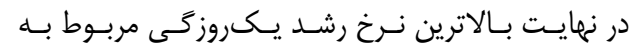

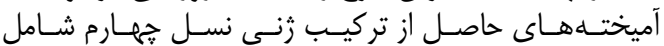
ABCD-DCBA

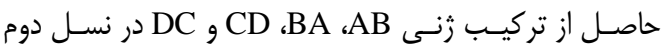

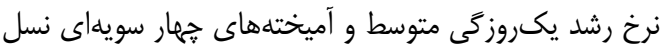

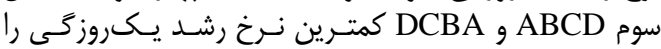

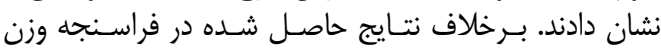

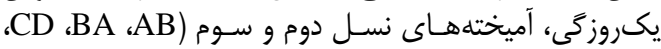

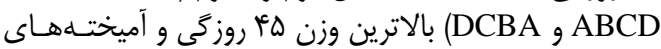

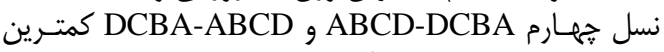

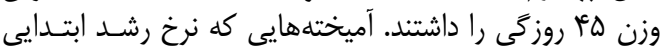

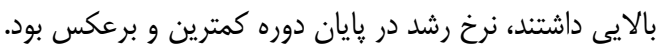

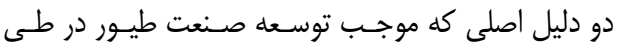

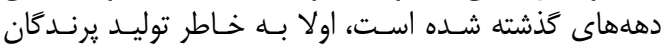

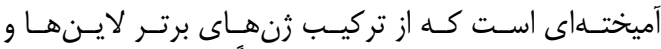

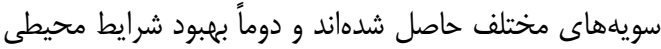

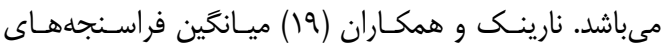

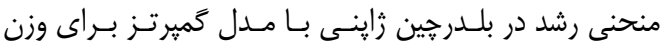

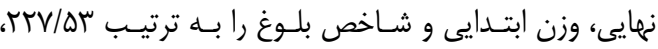

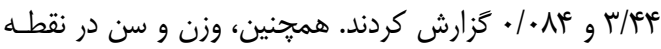

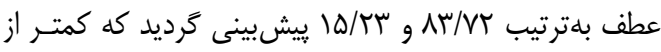

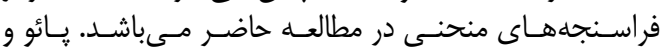

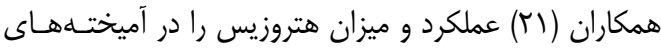

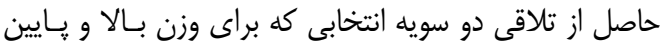

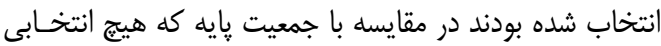

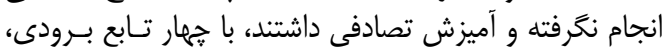

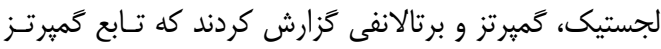

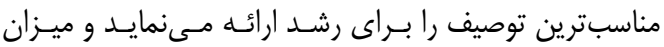

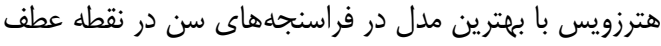

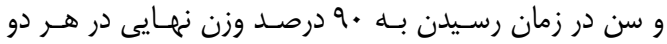

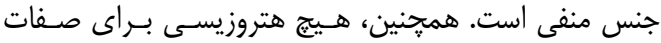


1. Aggrey, S.E. 2002. Comparison of three non-linear and spline regression models for describing chicken grow curves. Poultry Science, 81: 1782-1788.

2. Aggrey, S.E. 2003. Dynamic of relative growth rate in Japanese quail lines divergently selected for growth and their control. Growth Development and Aging, 67: 47-54.

3. Al-Murrani, W.K. 1978. Maternal effects on embryonic and post-embryonic growth in Poulttry. British Poultry Science, 19: 277-281

4. Arango, J.A. and L.D. Van Vleck. 2002. Size of beef cows; early ideas new developments. Genetic and Molecular Research 1: 51-63.

5. Bahreini Behzadi M.R., A.A. Aslaminejad, A.R. Sharifi and H. Simianer. 2014. Comparison of mathematical models for describing the growth of Baluchi sheep. Journal of Agriculture Science and Technology, 14: 57-68.

6. Balcioğlu, M.S., K. Kızılkaya, H. . Yolcu and H.I. Kabarağ. 2005. Analysis of growth characteristics in shortterm divergently selected Japanese quail. South African Journal of Animal Science, 35: 83-89.

7. Barbato, G.F. 1991. Genetic architecture of growth curve parameters in chickens. Theorical Applied Genetic, 83: 24-32.

8. Beiki, H., A. Pakdel, M. Moradi-shahrbabak and H. Mehrban. 2013. Evaluation of growth functions on Japanese quail lines. Journal of Poultry Science, 50: 20-27.

9. Brody, S. 1945. Bioenergetic and Growth. Reinhold Publishing Co, New York, USA, 1023 pp.

10. Darmani-Kuhi, H., T. Porter, S. Lopez, E. Kebreab, A.B. Strathe, A. Dumas, J. Dijkstra and J. France. 2010. A review of mathematical functions for the analysis of growth in poultry. World's Poultry Science Journal, 66: 227239.

11. Fair, J.M., E.S. Hansen and R.E. Ricklefs. 1999. Growth, developmental stability and immune response in juvenile Japanese quails. Proc. R. Soc London Ser B, 266: 1735-1742.

12. Firat, M.Z., E. Karaman, E.K. Başar and D. Narinc. 2016. Bayesian analysis for the comparison of nonlinear regression model parameters: an application to the growth of Japanese quail. Brazilian Journal of Poultry Science, 23: $19-26$.

13. Gompertz, B. 1825. On the nature of the function expressive of the law of human mortality, and on a new mode of determining the value of life contingencies. Philosophical transactions of the Royal Society of London, 115: 513-583.

14. Hashemirad, M., N. Farzin and A. Seraj. 2018. Genetic parameters of body weight and carcass characteristics in two strains of Japanese quail. Research on Animal Production, 8(17): 166-174 (In Persian).

15. Knizetova, H., J. Hyanek, B. Knize and J. Roubicek. 1991. Analysis of growth curves of fowl. I. Chickens. British Poultry Science, 32(5): 1027-1038

16. Loibel, S., M.G. Andrade, J.B. do Val and A.R.D. Freitas. 2010. Richards's growth model and viability indicators for populations subject to interventions. Anais da Academia Brasileira de Ciências, 82: 1107-1126.

17. Minvielle, F., D. Gourichon, S. Ito, M. Inoue-Murayama and S. Rivie. 2007. Effects of the dominant lethal yellow mutation on reproduction, growth, feed consumption, body temperature, and body composition of the Japanese quail. Poultry Science, 86: 1646-1650.

18. Nahashon, S.N., S.E. Aggrey, N.A. Adefope, A. Amenyenu and D. Wright. 2006. Growth characteristics of pearl gray guinea fowl as predicted by the Richards, Gompertz and logistic models. Poultry Science, 85(2): 359-363.

19. Narinc, D., E. Karaman, M.Z. Firat and T. Aksoy. 2010. Comparison of non-linear growth models to describe the growth in Japanese quail. Journal of Animal and Veterinary Advances, 9(14): 1961-1966.

20. Piao, J., S. Okamoto, S. Kobayashi, Y. Wada and Y. Maeda. 2002. Study of heterosis effects on productive traits of Japanese Quails: Heterosis effects on the crosses between large line and random bred population. Jpn Poultry Science, 39: J139-J146.

21. Piao, J., S. Okamoto, S. Kobayashi, Y. Wada and Y. Maeda. 2004. Purebred and crossbred performances from a Japanese quail line with very small body size. Animal Research, 53: 145-153.

22. Pinheiro, J., D. Bates, S. DebRoy and D. Sarkar. 2014. R Core Team nlme: linear and nonlinear mixed effects models. R package version 3.1-117. Available at h ttp://CRAN. R-project. org/package $=$ nlme.

23. Raji, A.O., S.T. Mbap and J. Aliyu. 2014. Comparison of different models to describe growth of the Japanese quail (Coturnix Japonica). Trakia Journal of Sciences, 2: 182-188.

24. Rezvannejad, E., A. Boustan and S. Lotfi. 2017. Comparison of reproductive performance of two pure lines of Japanese quail and their reciprocal crosses. Research on Animal Production, 8(15): 144-148 (In Persian).

25. Richards, F. 1959. A flexible growth function for empirical use. Journal of Experimental Botany, 10: 280-300.

26. Ricklefs R.E. 1985. Modification of growth and development of muscles in poultry. Poultry Science, 64: 15631576.

27. Robertson, T.B. 1908. On the normal rate of growth of an individual, and its biochemical significance. Archiv für Entwicklungsmechanik der Organismen, 25: 581-614.

28. Sekaninova, A., L. Kupcikova and M. Lichovnikova. 2016. The effect of divergent selection for shape of growth curve in Japanese quail on egg quality. MendelNet, 23: 269-272.

29. Sezer, M. and S. Tarhan. 2005. Function parameters of growth curves of three meat-type lines of Japanese quail. Czech Journal of Animal Science, 50: 22-30.

30. Taheri Dezfuli, B., M. Babaei, A. Kardooni. 2018. Fitting milk curve and its compounds for Khuzestani buffaloes using five different functions. Research on Animal Production, 9(19): 113-123 (In Persian).

31. Tarhyel, R., B.K. Tanimomo and S.A. Hena. 2012. Organ weight: As influenced by color, sex and weight group in Japanese quail. Scientific Journal of Animal Science, 1: 46-49.

32. Tariq, M.M., M.A. Bajwa, A. Waheed, E. Eyduran, F. Abbas, F.A. Bokhari and A. Akbar. 2011. Growth curve in Mengali sheep breed of Balochistan. Journal of Animal and Plant Science, 21: 5-7.

33. Verhulst, P.F. 1838. Notice Sur La Loi que la population suit dans son accroissement Correspondence Mathematique et Physique, 10: 113-121.

34. Vuori, K., I. Stranden, M.L. Sevon-Aimonen and E.A. Mantysaari. 2006. Estimation of non-linear growth models by linearization: a simulation study using a Gompertz function. Genetics Selection and Evolution, 38: 343-358.

35. Waheed, A., M. Sajjad Khan, S. Ali and M. Sarwar. 2011. Estimation of growth curve parameters in Beetal goats. Archiv Tierzucht, 54: 287-296.

36. Weibull, W. 1951. A statistical distribution function of wide applicability. Journal of Applied Mechanics, 12: 293-297. 


\title{
Growth Curves Fitting in Japanese Quail Di-Allele Crosses and Comparison of Growth Patterns of Crossbreds
}

\section{Khadijeh Ebrahimi ${ }^{1}$, Gholam Reza Dashab ${ }^{2}$, Hadi Faraji-Arough ${ }^{3}$ and Mohammad Rokouei ${ }^{4}$}

1 and 2- M.Sc. Student and Assistant Professor, of Animal Breeding, Department of Animal Science, Agriculture Faculty, University of Zabol

3- Assistant Professor, of Animal Breeding and Genetic, Research Center of Special Domestic Animals, University of Zabol (Corresponding author: hadifaraji@uoz.ac.ir)

4- Associate Professor of Animal Breeding, Department of Animal Science and Bioinformatics, Agriculture Faculty, University of Zabol

Received: October 31, 2017

Accepted: April 25, 2018

\begin{abstract}
The main part of growth performance in birds is as a result of gene combination effects, which their changes require an optimal mating system in order to use general and specific gene combination ability. The purpose of the present study was to evaluate the growth patterns of four strains combination Japanese quail in a partial di-allele cross. For this purpose, four strains of Italian Speckled (A), Tuxedo (B), Pharaoh (C), A\&M Texas (D) were used in a partial diallele cross design to produce different gene combinations during four generations. Four functions including Gomperts, Richards, Logistic and Weibull were fitted for body weights records of different combinations. The best function for each combination was selected by goodness of fit criteria's. Then, function parameters and growth patterns of the best function were compared between combinations. The results of goodness of fit criteria's showed that the Gompertz function was the best one for binary combinations of $\mathrm{AB}, \mathrm{BA}, \mathrm{CD}, \mathrm{DC}$ and reciprocal combination of ABCD-DCBA, DCBA-ABCD, while Weibul function was found to be the best function for quadrille combinations of $\mathrm{ABCD}$, DCBA for describing growth curve. The effects of year and month of hatching on all growth patterns were significant $(\mathrm{P}<0.05)$. Also, the combination effect on initial weight, final weight, age and weight at inflection point and, oneday weight was significant $(\mathrm{P}<0.05)$. The effect of sex on curve growth parameters was not significant $(\mathrm{P}>0.05)$ except for 45-days weight. The DC combination had the highest initial and final weight, age and weight at inflection point compared to other combinations. The quadrille combinations, especially DCBA had the highest final weight, 45-days weight and, 45-days growth rate, but the lowest puberty index. Also, age and weight at inflection point in DCBA combination were at moderate level among combinations and showed better performance compared to other crosses indicating of the most heterosis in this combination.
\end{abstract}

Keywords: Cross breeding, Growth function, Gompertz, Japanese quail 